\title{
Physical models of ten asteroids from an observers' collaboration network ${ }^{\star}$
}

\author{
J. Durech ${ }^{1,2}$, M. Kaasalainen ${ }^{2}$, A. Marciniak ${ }^{3}$, W. H. Allen ${ }^{23}$, R. Behrend ${ }^{22}$, C. Bembrick ${ }^{4}$, T. Bennett ${ }^{11}$, \\ L. Bernasconi ${ }^{5}$, J. Berthier ${ }^{25}$, G. Bolt ${ }^{6}$, S. Boroumand ${ }^{28}$, L. Crespo da Silva ${ }^{28}$, R. Crippa ${ }^{21}$, M. Crow ${ }^{7}$, R. Durkee $^{8}$, \\ R. Dymock ${ }^{9}$, M. Fagas ${ }^{3}$, M. Fauerbach ${ }^{11}$, S. Fauvaud ${ }^{10,29}$, M. Frey ${ }^{12}$, R. Gonçalves ${ }^{20}$, R. Hirsch ${ }^{3}$, D. Jardine ${ }^{15}$, \\ K. Kamiński ${ }^{3}$, R. Koff ${ }^{13}$, T. Kwiatkowski ${ }^{3}$, A. López ${ }^{14}$, F. Manzini ${ }^{21}$, T. Michałowski ${ }^{3}$, R. Pacheco ${ }^{14}$, M. Pan ${ }^{28}$, \\ F. Pilcher ${ }^{15}$, R. Poncy ${ }^{19}$, D. Pray ${ }^{16}$, W. Pych ${ }^{24}$, R. Roy ${ }^{17}$, G. Santacana ${ }^{10}$, S. Slivan ${ }^{12,28}$, S. Sposetti ${ }^{27}$, R. Stephens ${ }^{18}$, \\ B. Warner ${ }^{26}$, and M. Wolf ${ }^{1}$ \\ (Affiliations can be found after the references)
}

Received 5 September 2006 / Accepted 14 December 2006

\begin{abstract}
Aims. We present physical models of ten asteroids obtained by means of lightcurve inversion. A substantial part of the photometric data was observed by amateur astronomers. We emphasize the importance of a coordinated network of observers that will be of extreme importance for future all-sky asteroid photometric surveys.

Methods. The lightcurve inversion method was used to derive spin states and shape models of the asteroids.

Results. We derived spin states and shape model for ten new asteroids: (110) Lydia, (125) Liberatrix, (130) Elektra, (165) Loreley, (196) Philomela, (218) Bianca, (306) Unitas, (423) Diotima, (776) Berbericia, and (944) Hidalgo. This increases the number of asteroid models up to nearly one hundred.
\end{abstract}

Key words. minor planets, asteroids

\section{Introduction}

The lightcurve inversion method has become a standard tool for asteroid shape and spin state determination (Kaasalainen \& Torppa 2001; Kaasalainen et al. 2001, 2002a). Convex models are a good representation of real shapes of asteroids, as has been proven by ground truths from, e.g., Kaasalainen et al. (2001, 2005), and Marchis et al. (2006). Slightly fewer than one hundred asteroid models have been derived so far (Kaasalainen et al. 2002c, 2004; Torppa et al. 2003). However, the number of asteroid models increases slowly, mainly due to the fact that at least three well-covered apparitions are necessary for a main-belt asteroid to be modeled. The Uppsala Asteroid Photometric Catalogue (UAPC, Lagerkvist et al. 2001) - the main source of asteroid photometric data - has been already exploited and the global morphology of all well-observed asteroids has been determined. The UAPC still contains valuable photometric data of many asteroids, but the amount of the data is not sufficient for a unique physical model. For many such targets, observations from only one more apparition are sufficient for a model, and many of those targets are within the reach of amateur astronomers. We present new observations and physical models of asteroids (110) Lydia, (125) Liberatrix, (130) Elektra, (165) Loreley, (196) Philomela, (218) Bianca, (306) Unitas, (423) Diotima, (776) Berbericia, and (944) Hidalgo. In the last section, we discuss the possibility of combining the ordinary lightcurves with the sparse photometric data that will be available from all-sky photometric surveys in the near future.

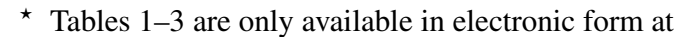
http: //www . aanda.org

\section{Observations}

To derive unique spin state solutions and shape models, we combined photometric data published in the UAPC with the new observations that were carried out by a large number of both amateur and professional observers. Some lightcurves from the UAPC that were too noisy, consisted of only a few points, or were clearly wrong were not included in the analysis. Information about observers and telescopes is listed in Table 1. All the new observations are listed in Table 2. For each lightcurve, there is the date of observation, the aspect data, the asteroid's ecliptic coordinates, and the code number of the observatory referring to Table 1 . All photometric observations were treated as relative and we used a combination of the Lommel-Seeliger and Lambert light-scattering laws (Kaasalainen et al. 2002a) as our scattering model.

We do not present all the lightcurves in a graphical form, but only a selection of three representative lightcurves for each asteroid. Most of the lightcurves from Table 2 can be found at the Collaborative Asteroid Lightcurve Link (http://www. MinorPlanetObserver.com/ astlc/default.htm) or at http://obswww.unige.ch/ ־behrend/page_cou.html. The complete lightcurve datasets that were used for deriving shape models in the following section can be downloaded from http://cdsweb.u-strasbg.fr/ cgi-bin/qcat?J/A+A/465/331.

\section{Models}

The spin solutions and shape models were derived using the lightcurve inversion method developed by Kaasalainen \& Torppa (2001), and Kaasalainen et al. (2001). The spin axis 

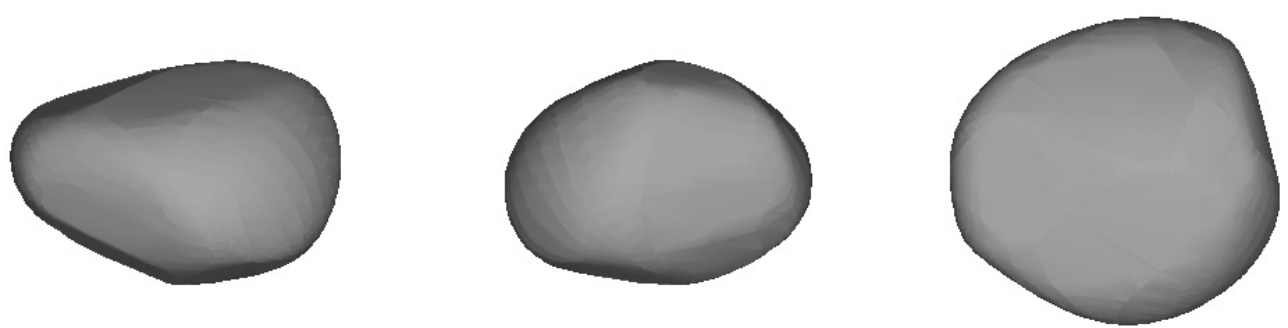

Fig. 1. The shape model of (110) Lydia. There are two equatorial views $90^{\circ}$ apart (the first two figures) and a pole-on view (the third plot).
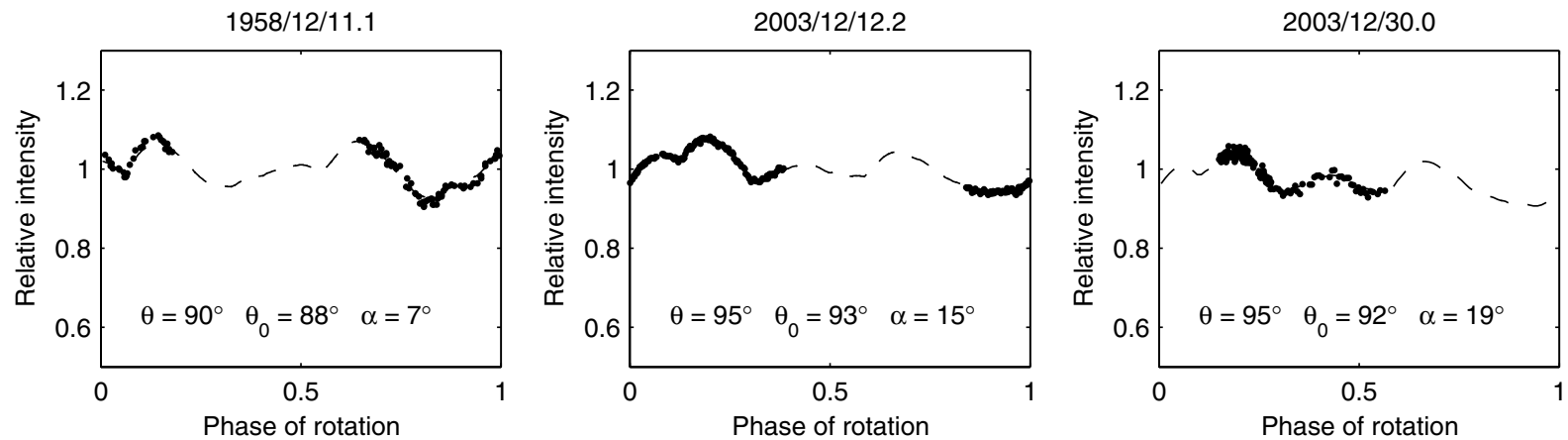

Fig. 2. Observed data (points) and the modeled brightness (dashed curve) for three representative lightcurves of (110) Lydia. The plots cover one rotation cycle, the brightness is given in relative intensity units. The viewing/illumination geometry is given by the aspect angle of the Earth $\theta$ and of the Sun $\theta_{0}$ and by the solar phase angle $\alpha$.
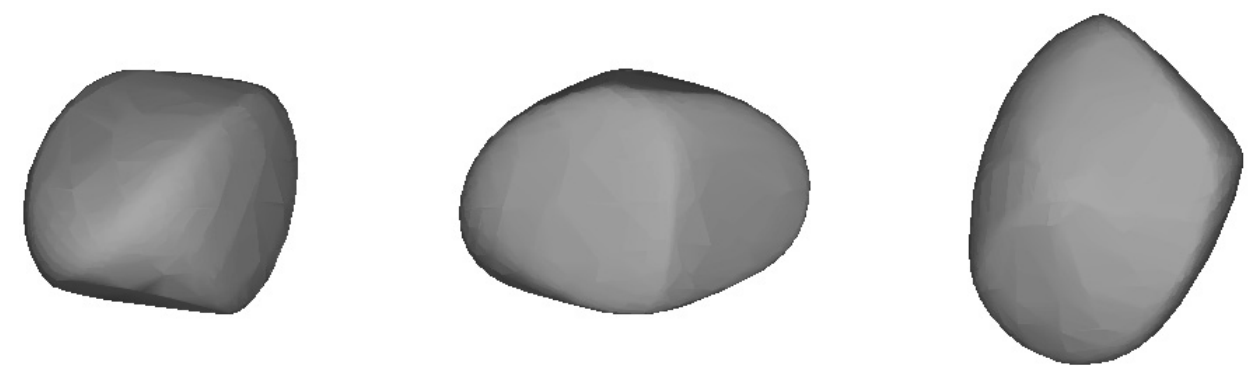

Fig. 3. The shape model of (125) Liberatrix. The viewing geometry is the same as in Fig. 1.
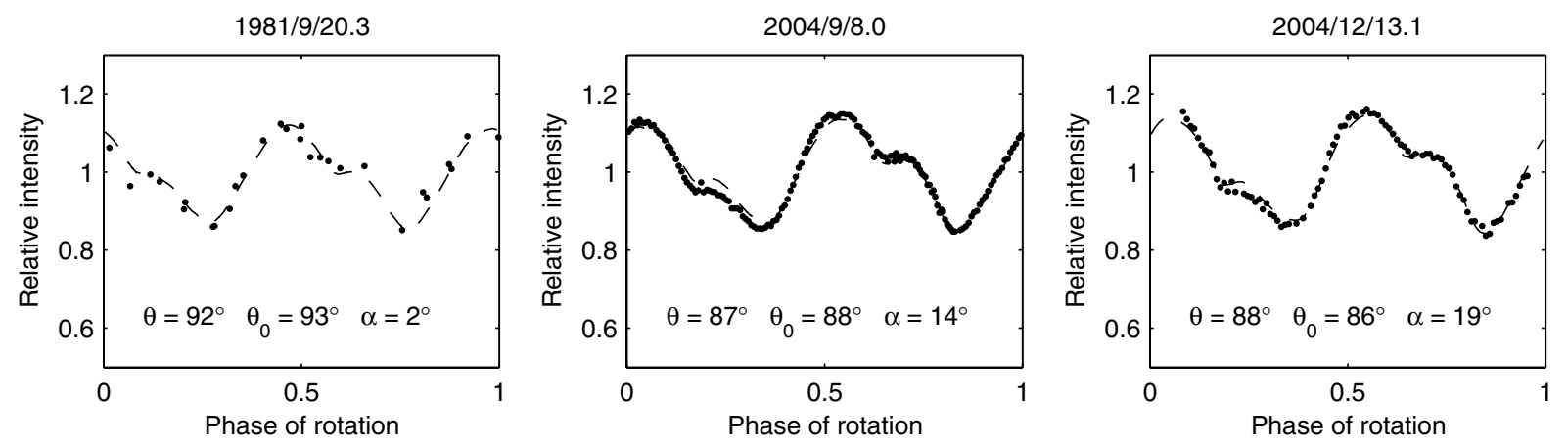

Fig. 4. Lightcurves and the corresponding fits for (125) Liberatrix.

direction in $\mathrm{J} 2000$ ecliptic coordinates $\lambda_{\mathrm{p}}, \beta_{\mathrm{p}}$ and the rotation period $P$ for each asteroid are listed in Table 3 . In the case of the lightcurve inversion, the systematic errors in lightcurves and model errors dominate over the observational noise. Thus formal errors for pole positions derived from confidence limits based on $\chi^{2}$ distribution would be underestimated. A good estimation of a typical error in the pole direction obtained by comparison of lightcurve inversion results with ground truths from space probes and laboratory experiments (Kaasalainen et al. 2005) is about $5^{\circ}$ of arc. The accuracy of the period determination is of the order of the last unit digit of the period value given in Table 3. For more detailed discussion of error estimation see Torppa et al. (2003), and Kaasalainen \& Ďurech (2007).

In Figs. 1 to 20, we plot the the shape model of each asteroid viewed from the plane of its equator (two views $90^{\circ}$ apart) and pole-on, and the corresponding lightcurve fit. In some cases, there are two possible pole solutions with the ecliptic longitudes $\lambda$ about $180^{\circ}$ apart and with similar values 

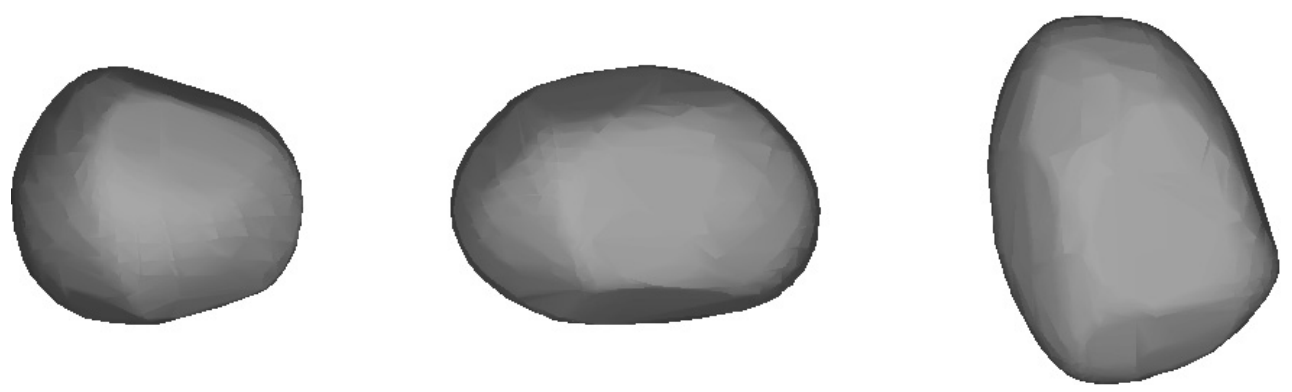

Fig. 5. The shape model of (130) Elektra. The viewing geometry is the same as in Fig. 1.
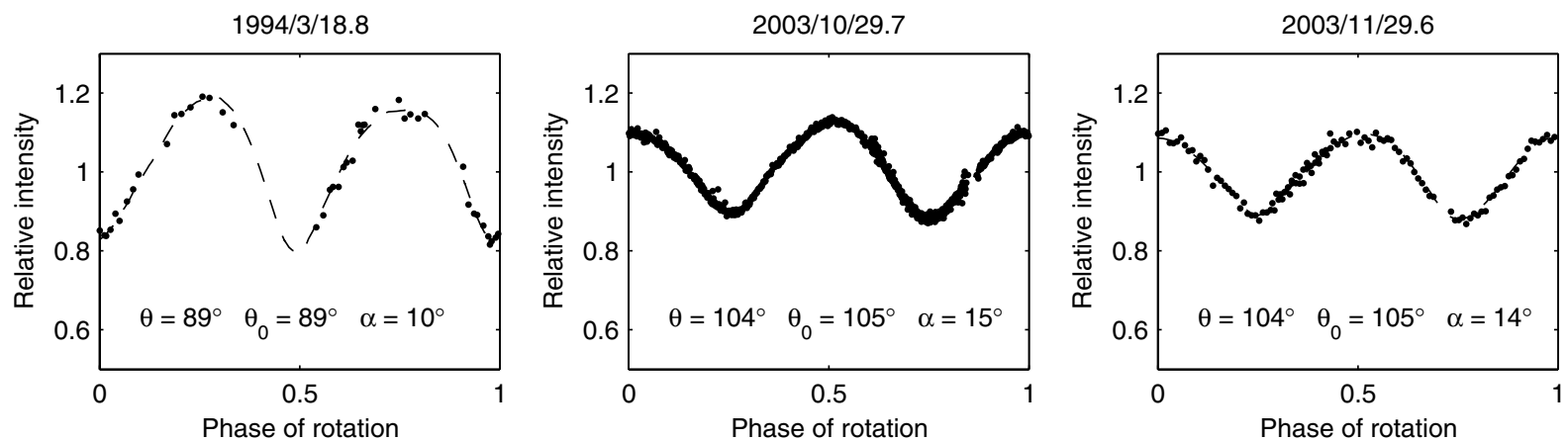

Fig. 6. Lightcurves and the corresponding fits for (130) Elektra.
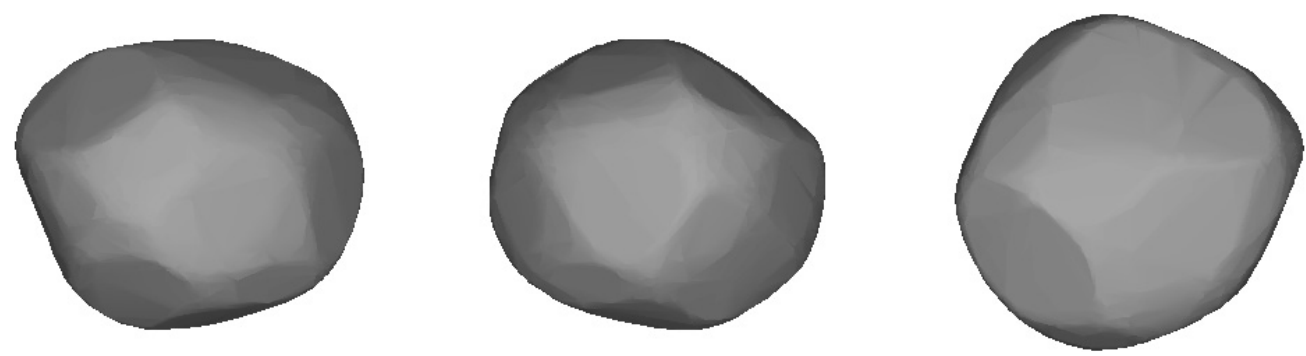

Fig. 7. The shape model of (165) Loreley. The viewing geometry is the same as in Fig. 1.
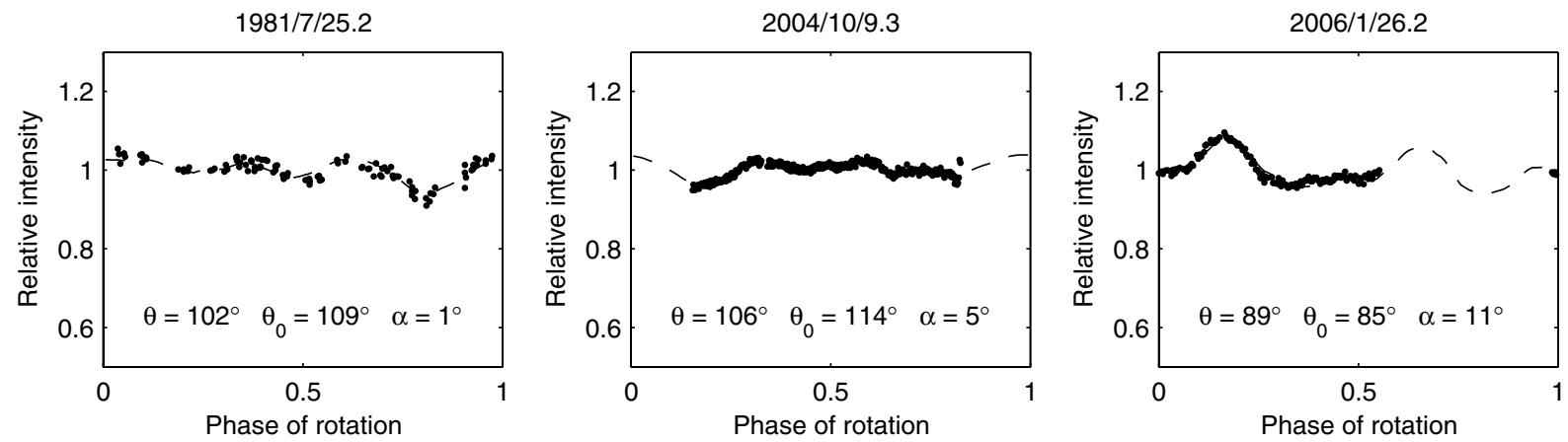

Fig. 8. Lightcurves and the corresponding fits for (165) Loreley.
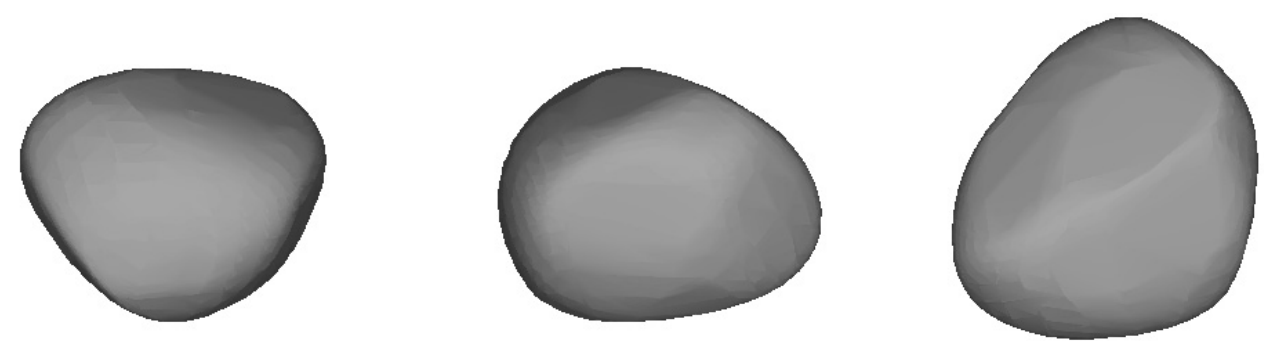

Fig. 9. The shape model of (196) Philomela. The viewing geometry is the same as in Fig. 1. 

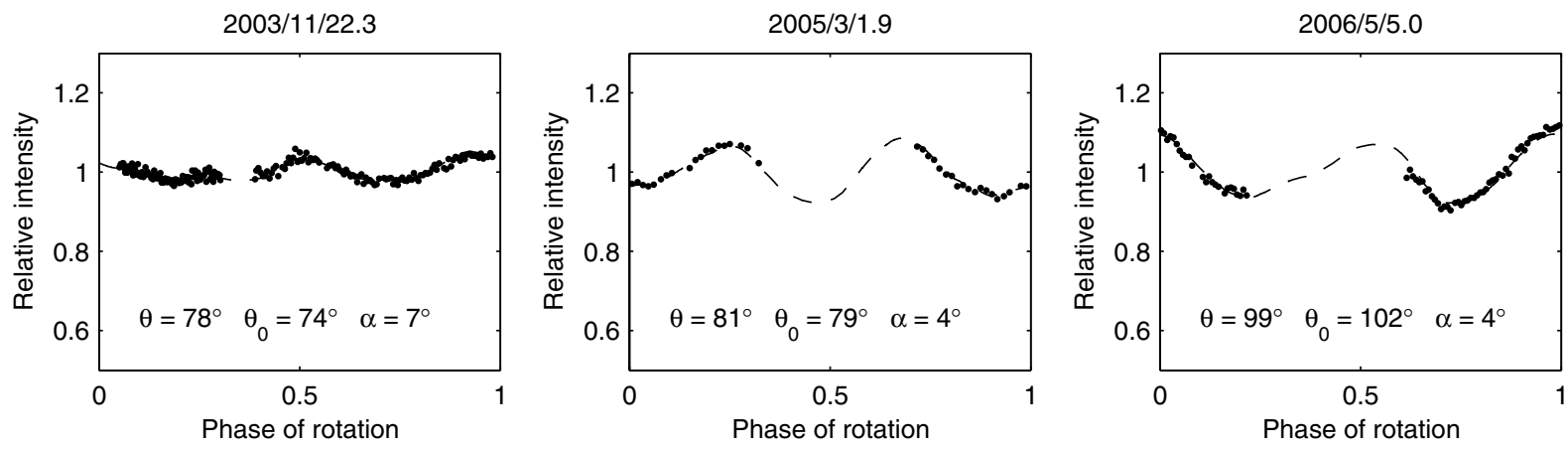

Fig. 10. Lightcurves and the corresponding fits for (196) Philomela.
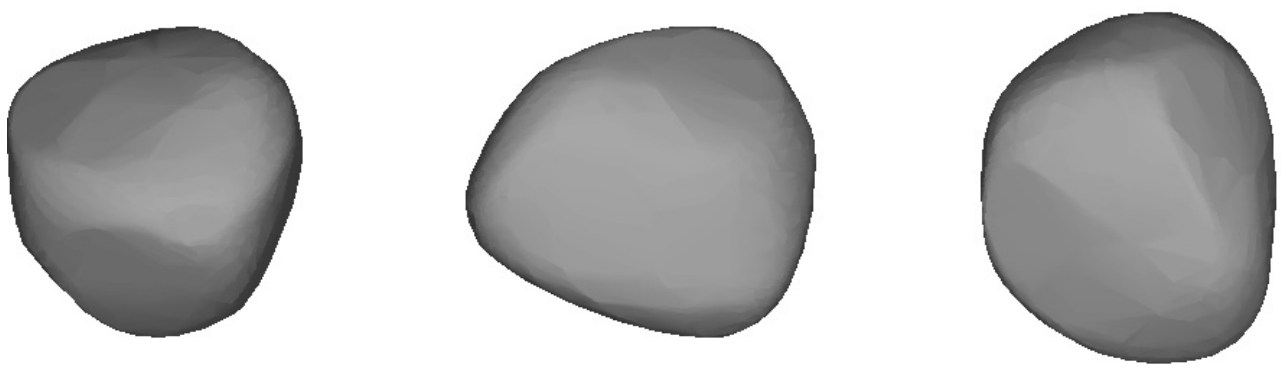

Fig. 11. The shape model of (218) Bianca. The viewing geometry is the same as in Fig. 1.
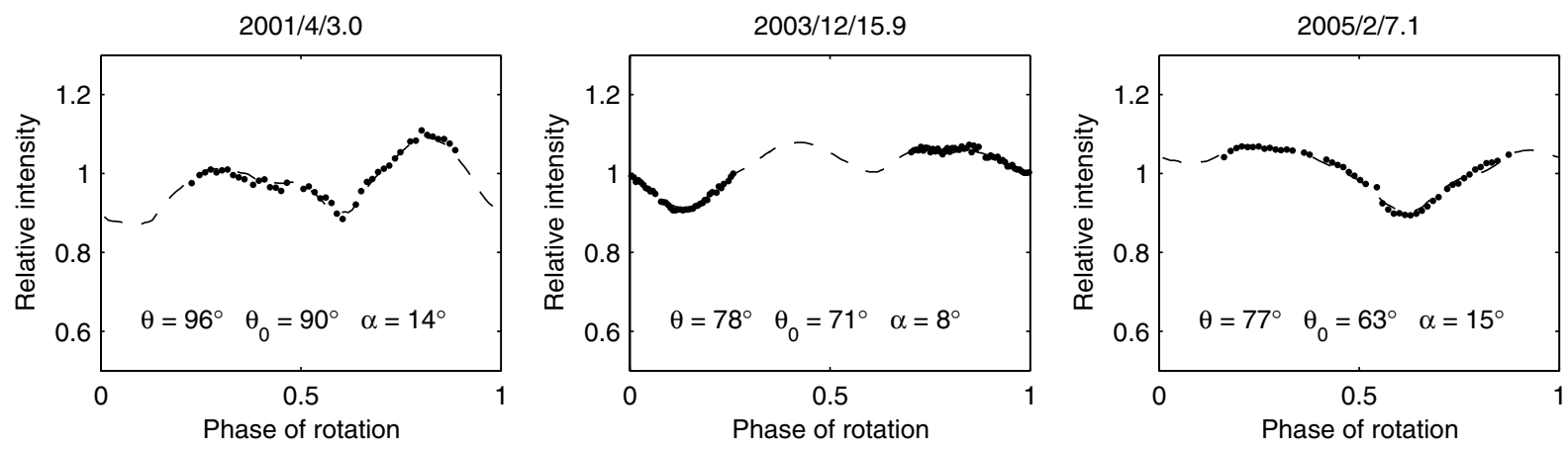

Fig. 12. Lightcurves and the corresponding fits for (218) Bianca.
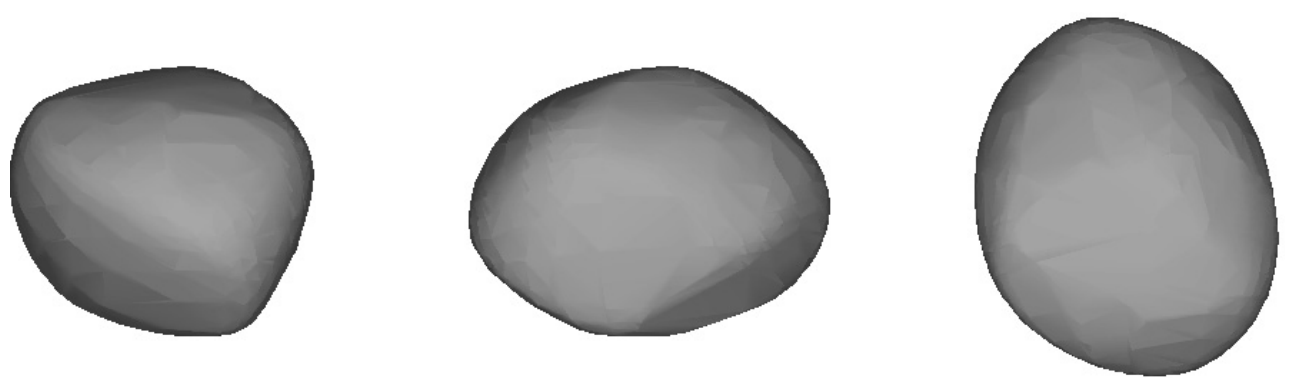

Fig. 13. The shape model of (306) Unitas. The viewing geometry is the same as in Fig. 1.

of the pole ecliptic latitude $\beta$. This ambiguity is inevitable for disk-integrated measurements of objects orbiting near the plane of ecliptic (see Kaasalainen \& Lamberg 2006). Due to the fact that we used only relative photometry, the dimensions along the rotation axis are not well constrained. The pole-on silhouettes are very good approximations of asteroids' real shapes, whereas the silhouettes viewed from the equatorial plane can be significantly stretched or squeezed along the rotation axis. The principal axis of the inertia tensor (assuming uniform density) corresponding to the maximum moment of inertia is very close to the rotation axis for every model. The models together with the spin vector solutions are available at http://astro.troja.mff.cuni.cz/projects/ asteroids3D.

(110) Lydia. Lightcurve amplitudes do not exceed 0.2 mag. The shape is flat with a regular pole-on silhouette. There are two pole solutions.

(125) Liberatrix. The rotation axis is almost perpendicular to the ecliptic plane and the orbit is close to the ecliptic (for most 

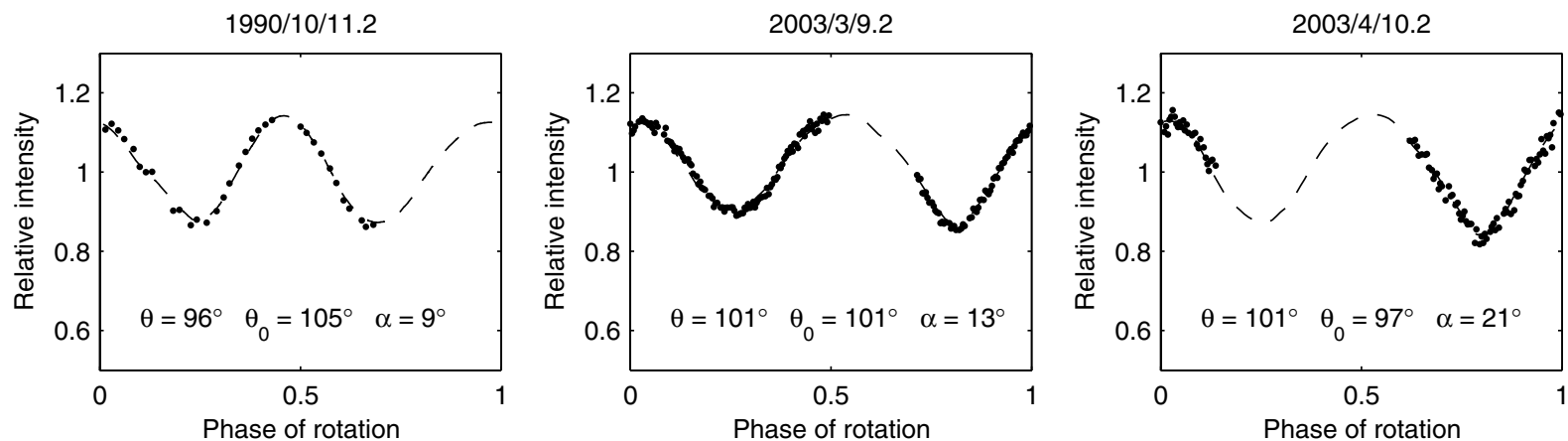

Fig. 14. Lightcurves and the corresponding fits for (306) Unitas.
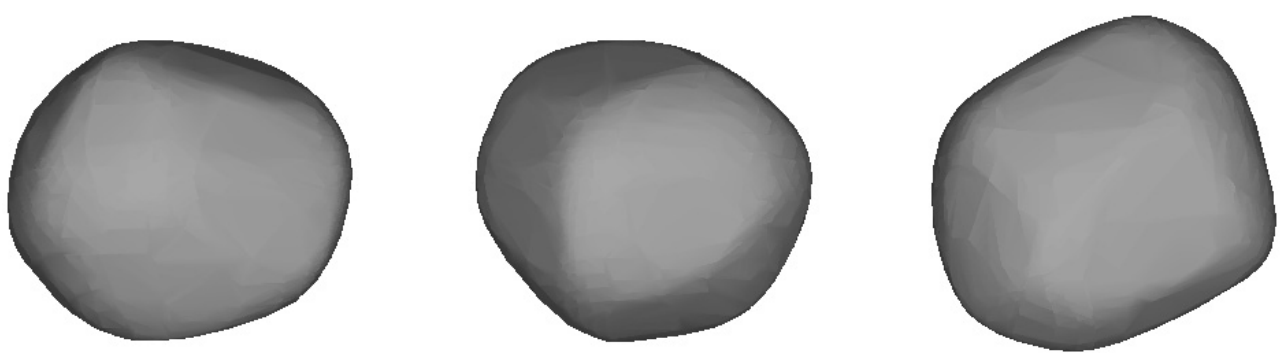

Fig. 15. The shape model of (423) Diotima. The viewing geometry is the same as in Fig. 1.
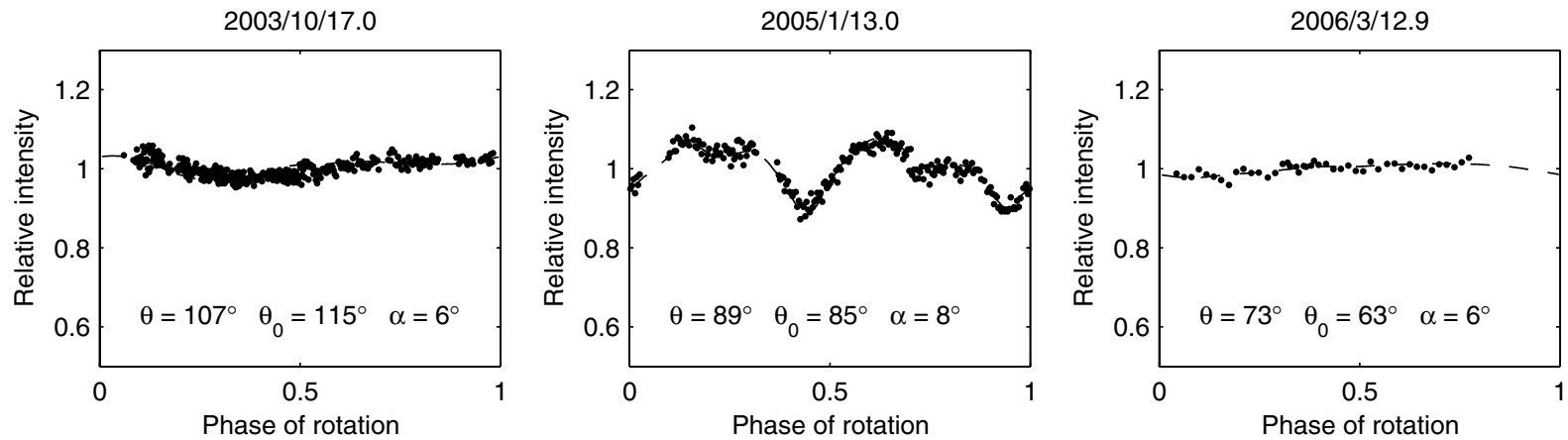

Fig. 16. Lightcurves and the corresponding fits for (423) Diotima.
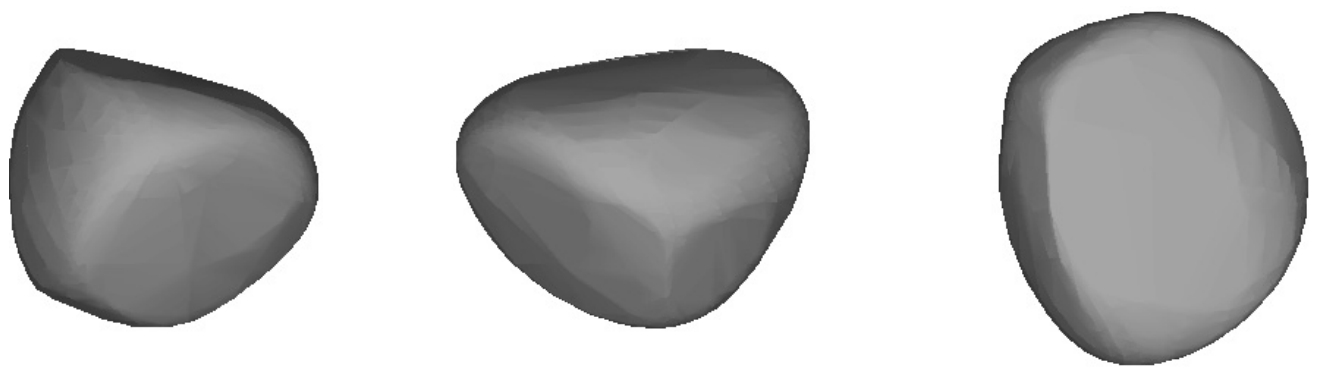

Fig. 17. The shape model of (776) Berbericia. The viewing geometry is the same as in Fig. 1.

lightcurves $|\beta| \leq 5^{\circ}$ ). Liberatrix has been seen equator-on all the time and the lightcurves hardly change from one opposition to another, having the same amplitude $0.4 \mathrm{mag}$. Relative lightcurves and the restricted geometry do not allow us to constrain the dimension along the rotation axis accurately - the shape model can be more or less stretched along this axis and the lightcurve fits remain almost the same.

(130) Elektra. Lightcurves of Elektra are typical double sinusoidal, the shape model is regular and elongated. Although the rotation axis is perpendicular to the plain of ecliptic, the viewing/illumination geometry is not restricted to the equatorial view/illumination (contrary to the previous case of Liberatrix), due to the high ecliptic latitude Elektra reached $\left(-35^{\circ}<\beta<25^{\circ}\right)$.

(165) Loreley. The shape model has many flat areas, the lightcurves have small amplitudes of 0.2 mag at most and a complicated structure. The pole direction solution $\left(346^{\circ},+29^{\circ}\right)$ is 

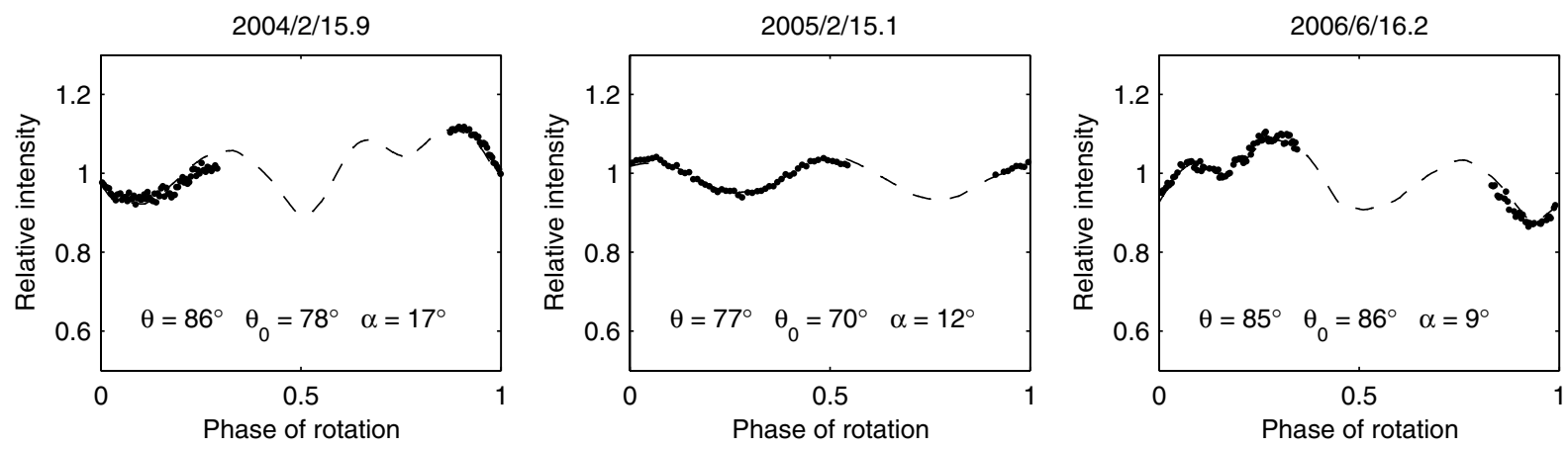

Fig. 18. Lightcurves and the corresponding fits for (776) Berbericia.
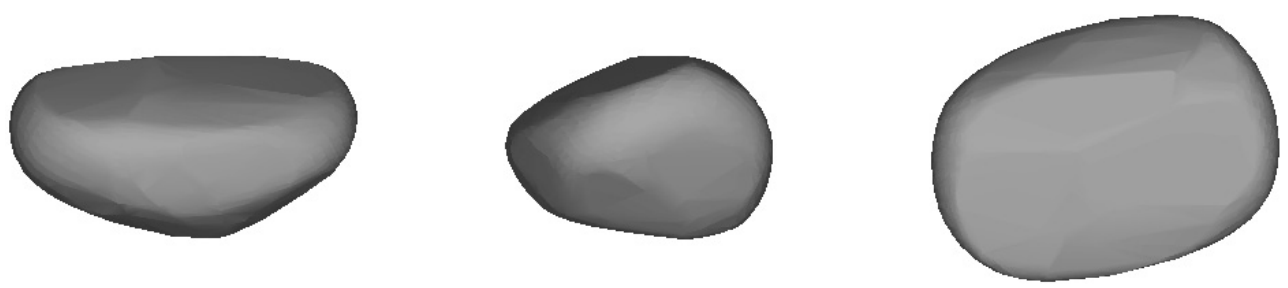

Fig. 19. The shape model of (944) Hidalgo. The viewing geometry is the same as in Fig. 1.
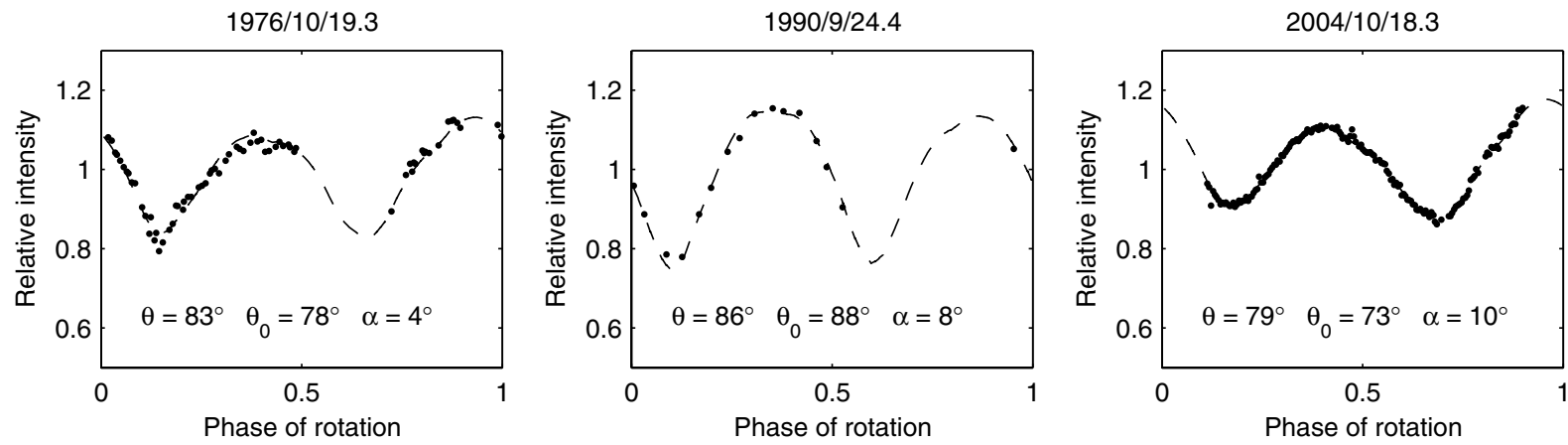

Fig. 20. Lightcurves and the corresponding fits for (944) Hidalgo.

clearly the best one, but there is the second solution $\left(165^{\circ},+15^{\circ}\right)$ giving only a slightly worse fit.

(196) Philomela. The shape model is asymmetric and smooth, the geometry varies a lot, lightcurves vary from almost flat to those with amplitudes up to $0.4 \mathrm{mag}$.

(218) Bianca. There are two pole solutions corresponding to almost the same spin axis with both a prograde and a retrograde sense of rotation. The shape is asymmetric.

(306) Unitas. The shape is regular, lightcurves typically exhibit two extrema per rotation.

(423) Diotima. The lightcurves vary a lot - some are almost flat and others exhibit 0.2 mag deep minima. From the photometric data we obtained two solutions for the pole direction: $\left(351^{\circ},+4^{\circ}\right)$ and $\left(176^{\circ},+33^{\circ}\right)$, but only the first one is consistent with the adaptive optics image obtained by Marchis et al. (2006).
(776) Berbericia. Lightcurves are very different for different apparitions - sometimes there is only one maximum per period. The corresponding shape model is asymmetric with sharp edges.

(944) Hidalgo. Although our model is based on only 14 lightcurves from four oppositions, the pole and period solution is unique. The shape model has very large flat areas and a "rectangular" pole-on silhouette, which are strong indications of a highly nonconvex shape (Kaasalainen et al. 2002b; Durech \& Kaasalainen 2003). Also, the sharp minima of some lightcurves support the idea of a two-lobed shape.

\section{Future work}

The number of asteroid models available so far is very small when compared with the whole asteroid population. The classical approach of observing a selected target (or a few targets) during the night to densely cover the lightcurve in the rotation phase is time consuming. The number of asteroids with enough observations to derive a model increases only slowly. The situation is going to change in the near future with the asteroid photometric surveys (for example Pan-STARRS). It has been shown (see Kaasalainen 2004; Durech et al. 2005) that asteroid models can be derived from calibrated photometric measurements that are 
sparse in time. This kind of data will be provided by future photometric surveys - instead of tens of lightcurves covering several apparitions we will have typically a hundred or more individual brightness measurements spread over several years. A difficulty that appears when analyzing sparse data is that the rotation period of an asteroid is not "visible" from the data as it is in the case of an ordinary well-covered lightcurve. Thus a very wide interval of all possible periods must be densely scanned for the correct value. The time-consuming process of period search can be sped up dramatically by adding just one ordinary lightcurve that constrains the search to a narrow interval of periods. A network of observers who will carry out photometric follow-up observations of selected asteroids will be very important for the next generation of all-sky surveys.

Acknowledgements. This work was supported, in part, by CIMO and the Academy of Finland. The observations carried out at the Borowiec Station were supported by Polish Grant 1 P03D 02027.

\section{References}

Ďurech, J., \& Kaasalainen, M. 2003, A\&A, 404, 709

Durech, J., Grav, T., Jedicke, R., Kaasalainen, M., \& Denneau, L. 2005, Earth, Moon, and Planets, 97, 179

Kaasalainen, M. 2004, A\&A, 422, L39

Kaasalainen, M., \& Torppa, J. 2001, Icarus, 153, 24

Kaasalainen, M., \& Lamberg, L. 2006, Inverse Problems, 22, 749

Kaasalainen, M., \& Durech, J. 2007, in Near Earth Objects, our Celestial Neighbors: Opportunity and Risk, ed. A. Milani, G. B. Valsecchi, \& D. Vokrouhlický (Cambridge: Cambridge University Press), in press

Kaasalainen, M., Torppa, J., \& Muinonen, K. 2001, Icarus, 153, 37

Kaasalainen, M., Mottola, S., \& Fulchignomi, M. 2002a, in Asteroids III, ed. W. F. Bottke, A. Cellino, P. Paolicchi, \& R. P. Binzel (Tucson: University of Arizona Press), 139

Kaasalainen, M., Torppa, J., \& Piironen, J. 2002b, A\&A, 383, L19

Kaasalainen, M., Torppa, J., \& Piironen, J. 2002c, Icarus, 159, 369

Kaasalainen, M., Pravec, P., Krugly, Y. N., et al. 2004, Icarus, 167, 178

Kaasalainen, S., Kaasalainen, M., \& Piironen, J. 2005, A\&A, 440, 1177

Lagerkvist, C.-I., Piironen, J., \& Erikson, A. 2001, Asteroid photometric catalogue, fifth update (Uppsala Astronomical Observatory)

Marchis, F., Kaasalainen, M., Hom, E. F. Y., et al. 2006, Icarus, 185, 39

Torppa, J., Kaasalainen, M., Michalowski, T., et al. 2003, Icarus, 164, 346

1 Astronomical Institute, Charles University in Prague V Holešovičkách 2, 18000 Prague, Czech Republic

e-mail: durech@sirrah.troja.mff.cuni.cz

2 Department of Mathematics and Statistics, Rolf Nevanlinna Institute, University of Helsinki, PO Box 68, 00014 Helsinki, Finland

3 Astronomical Observatory, A. Mickiewicz University, Słoneczna 36, 60-286 Poznań, Poland
4 Mt Tarana Observatory, PO Box 1537, Bathurst, NSW 2795, Australia

5 Observatoire des Engarouines, 84570 Mallemort-du-Comtat, France

6295 Camberwarra Drive, Craigie, WA 6025, Australia

7 The WW Crow Observatory, 118 Mill Road, Hawley, Kent, DA2 7RT, England

8 Shed of Science Observatory, 5213 Washburn Ave. S., Minneapolis, MN 55410, USA

${ }^{9} 67$ Haslar Crescent, Waterlooville, Hampshire, PO7 6DD, England

10 Association AstroQueyras, Le Bois de Bardon, 16110 Taponnat, France

11 Florida Gulf Coast University, 10501 FGCU Boulevard South, Fort Myers, FL 33965, USA

12 Department of Astronomy, Whitin Observatory, Wellesley College, 106 Central Street, Wellesley, MA 02481, USA

13 Antelope Hills Observatory, 980 Antelope Drive West, Bennett, CO 80102, USA

${ }^{14}$ Observatori Astronomic de Consell, Mallorca, Spain

15 Illinois College, 1101 West College Avenue, Jacksonville, IL 62650, USA

16 Carbuncle Hill Observatory, PO Box 946, Coventry, RI 02816, USA

17 Observatoire de Blauvac, 84570 St-Estève, France

18 Goat Mountain Astronomical Research Station, 11355 Mount

Johnson Court, Rancho Cucamonga, CA 91737, USA

192 rue des Écoles 34920, Le Crès, France

20 Linhaceira Observatory, Instituto Politècnico de Tomar, 2300-

313, Tomar, Portugal

21 Stazione Astronomica di Sozzago, 28060 Sozzago, Italy

22 Geneva Observatory, 1290 Sauverny, Switzerland

23 Vintage Lane Observatory, 83 Vintage Lane, RD3, Blenheim, New Zealand

${ }_{24}$ Copernicus Astronomical Center, Bartycka 18, 00-716 Warszawa, Poland

${ }^{25}$ Institut de mécanique céleste et de calcul des éphémérides, Observatoire de Paris, 77 Av. Denfert Rochereau, 75014 Paris, France

${ }^{26}$ Palmer Divide Observatory, 17995 Bakers Farm Rd., Colorado Springs, CO 80908, USA

${ }^{27}$ Observatorio di Gnosca, 6525 Gnosca, Switzerland

28 Dept. of Earth, Atmospheric, and Planetary Sciences, Massachusetts Institute of Technology, 77 Massachusetts Ave., Cambridge, MA 02139, USA

${ }^{29}$ Groupe Européen d'Observations Stellaires (GEOS), 23 Parc de Levesville, 28300 Bailleau l'Evêque, France 
J. Ďurech et al.: Physical models of ten asteroids, Online Material p 1

\section{Online Material}


Table 1. The list of observatories and telescopes, $D$ is the telescope aperture diameter.

\begin{tabular}{rlcl}
\hline \hline Code & Observing site & $D$ [cm] & Observers \\
\hline 1 & Blauvac Observatory, France & 31 & R. Roy \\
2 & Borowiec Station, Poznań Observatory, Poland & 40 & A. Marciniak, R. Hirsch, K. Kamiński, \\
3 & Carbuncle Hill Observatory, Rhode Island, USA & 35 & M. Fagas, T. Michałowski, T. Kwiatkowski \\
4 & Egan Observatory, Florida, USA & 40 & M. Fauerbach, T. Bennett \\
5 & Observatori Astronomic de Consell, Mallorca, Spain & 40 & A. Lopez, R. Pacheco \\
7 & Ondřejov Observatory, Czech Republic & 65 & M. Wolf, J. Durech \\
8 & Ostrowik, Poland & 60 & W. Pych \\
9 & Pic du Midi, France & 105 & T. Michałowski, J. Berthier \\
11 & Santana Observatory, CA, USA & 35 & R. Stephens \\
12 & Shed of Science Observatory, Minneapolis, USA & 25 & R. Durkee \\
13 & Pic de Chateau-Renard Observatory, France & 62 & S. Fauvaud, G. Santacana \\
14 & Waterlooville, Hampshire, England & 25 & R. Dymock \\
15 & Whitin Observatory, Massachusetts, USA & 61 & S. Slivan, M. Frey \\
16 & Mt Tarana Observatory, Bathurst, Australia & 40 & C. Bembrick \\
17 & Craigie, Australia & 25 & G. Bolt \\
18 & Pleasant Plains, Illinois, USA & 35 & F. Pilcher, D. Jardine \\
19 & Le Crès, France & 25 & R. Poncy \\
20 & Linhaceira Observatory, Portugal & 25 & R. Gonçalves \\
21 & Stazione Astronomica di Sozzago, Italy & 40 & R. Crippa, F. Manzini \\
22 & Vintage Lane Observatory, Blenheim, New Zealand & 30 & W. H. Allen \\
23 & The WW Crow Observatory, England & 20 & M. Crow \\
24 & Antelope Hills Observatory, Colorado, USA & 25 & R. Koff \\
25 & Les Engarouines Observatory, France & 21 & L. Bernasconi \\
26 & Goat Mountain Astronomical Research Station, USA & 35 & R. Stephens \\
27 & Gnosca Observatory, Switzerland & 40 & S. Sposetti \\
28 & Wallace Astrophysical Observatory, Massachusetts, USA & 61 & S. Slivan \\
29 & Wallace Astrophysical Observatory, Massachusetts, USA & 35 & M. Pan, L. Crespo da Silva, S. Boroumand \\
\hline
\end{tabular}


J. Ďurech et al.: Physical models of ten asteroids, Online Material p 3

Table 2. Aspect data for new observation of the modeled asteroids. The table lists asteroid distance from the Sun $r$, from the Earth $\Delta$, the solar phase angle $\alpha$, and the ecliptical coordinates of the asteroid $(\lambda, \beta)$.

\begin{tabular}{|c|c|c|c|c|c|c|}
\hline$\overline{\overline{\text { Date }}}$ & $\begin{array}{c}r \\
{[\mathrm{AU}]}\end{array}$ & $\begin{array}{c}\Delta \\
{[\mathrm{AU}]}\end{array}$ & $\begin{array}{c}\alpha \\
{[\mathrm{deg}]}\end{array}$ & $\begin{array}{c}\lambda \\
{[\mathrm{deg}]}\end{array}$ & $\begin{array}{c}\beta \\
{[\mathrm{deg}]}\end{array}$ & $\begin{array}{l}\text { Obs. } \\
\text { code }\end{array}$ \\
\hline \multicolumn{7}{|c|}{ (110) Lydia } \\
\hline 20031202.2 & 2.642 & 1.757 & 11.6 & 37.0 & -1.3 & 3 \\
\hline 20031213.1 & 2.651 & 1.857 & 15.1 & 35.9 & -0.9 & 3 \\
\hline 20031221.2 & 2.657 & 1.947 & 17.2 & 35.7 & -0.6 & 3 \\
\hline 20031222.0 & 2.658 & 1.957 & 17.4 & 35.7 & -0.6 & 3 \\
\hline 20031223.1 & 2.659 & 1.969 & 17.6 & 35.7 & -0.5 & 3 \\
\hline 20031226.1 & 2.661 & 2.007 & 18.3 & 35.8 & -0.4 & 3 \\
\hline 20031227.1 & 2.662 & 2.018 & 18.5 & 35.8 & -0.4 & 3 \\
\hline 20031228.0 & 2.663 & 2.029 & 18.6 & 35.8 & -0.4 & 3 \\
\hline 20031229.1 & 2.663 & 2.042 & 18.8 & 35.9 & -0.3 & 3 \\
\hline 20031230.0 & 2.664 & 2.055 & 19.0 & 35.9 & -0.3 & 3 \\
\hline 20031212.2 & 2.650 & 1.848 & 14.9 & 36.0 & -0.9 & 11 \\
\hline 20031217.2 & 2.654 & 1.901 & 16.3 & 35.8 & -0.7 & 11 \\
\hline 20031219.2 & 2.656 & 1.923 & 16.8 & 35.7 & -0.7 & 11 \\
\hline 20031222.2 & 2.658 & 1.958 & 17.5 & 35.7 & -0.6 & 11 \\
\hline \multicolumn{7}{|c|}{ (125) Liberatrix } \\
\hline 20041213.0 & 2.818 & 2.278 & 18.7 & 14.7 & -4.0 & 4 \\
\hline 20041213.1 & 2.818 & 2.279 & 18.7 & 14.7 & -4.0 & 4 \\
\hline 20041215.0 & 2.820 & 2.305 & 18.9 & 14.9 & -4.0 & 4 \\
\hline 20041228.0 & 2.830 & 2.489 & 20.0 & 16.4 & -3.9 & 4 \\
\hline 20041228.1 & 2.830 & 2.490 & 20.0 & 16.4 & -3.9 & 4 \\
\hline 20040908.0 & 2.740 & 1.907 & 14.2 & 27.5 & -2.7 & 7 \\
\hline 20041008.0 & 2.765 & 1.773 & 3.0 & 22.3 & -3.7 & 7 \\
\hline 20041230.7 & 2.832 & 2.529 & 20.1 & 16.9 & -3.9 & 7 \\
\hline 20050113.8 & 2.842 & 2.734 & 20.2 & 19.6 & -3.7 & 7 \\
\hline 20041110.1 & 2.792 & 1.905 & 11.0 & 15.7 & -4.2 & 15 \\
\hline \multicolumn{7}{|c|}{ (130) Elektra } \\
\hline 20010422.9 & 3.705 & 2.817 & 8.4 & 226.1 & 29.9 & 27 \\
\hline 20010423.0 & 3.705 & 2.817 & 8.4 & 226.1 & 29.9 & 27 \\
\hline 20031021.8 & 2.503 & 1.701 & 16.4 & 59.4 & -34.4 & 17 \\
\hline 20031023.7 & 2.505 & 1.694 & 16.1 & 59.1 & -34.7 & 17 \\
\hline 20031024.7 & 2.506 & 1.690 & 15.9 & 58.9 & -34.8 & 17 \\
\hline 20031029.7 & 2.511 & 1.676 & 15.0 & 58.0 & -35.4 & 17 \\
\hline 20031114.5 & 2.528 & 1.662 & 13.4 & 54.5 & -36.2 & 16 \\
\hline 20031115.6 & 2.530 & 1.663 & 13.4 & 54.2 & -36.2 & 16 \\
\hline 20031119.5 & 2.534 & 1.669 & 13.4 & 53.3 & -36.2 & 16 \\
\hline 20031127.6 & 2.544 & 1.692 & 13.8 & 51.5 & -35.8 & 16 \\
\hline 20031129.6 & 2.547 & 1.700 & 14.0 & 51.0 & -35.6 & 16 \\
\hline \multicolumn{7}{|c|}{ (165) Loreley } \\
\hline 20040928.3 & 3.064 & 2.102 & 6.3 & 17.7 & 15.6 & 12 \\
\hline 20041009.2 & 3.073 & 2.100 & 5.1 & 15.4 & 15.8 & 12 \\
\hline 20041009.3 & 3.073 & 2.100 & 5.1 & 15.4 & 15.8 & 12 \\
\hline 20041023.9 & 2.778 & 1.799 & 4.5 & 18.8 & -4.0 & 1 \\
\hline 20041105.9 & 2.789 & 1.872 & 9.5 & 16.4 & -4.1 & 1 \\
\hline 20041106.9 & 2.790 & 1.880 & 9.9 & 16.2 & -4.1 & 1 \\
\hline 20041110.0 & 2.792 & 1.904 & 10.9 & 15.8 & -4.2 & 1 \\
\hline 20041110.9 & 2.793 & 1.912 & 11.3 & 15.6 & -4.2 & 1 \\
\hline 20060115.2 & 3.365 & 2.482 & 8.6 & 84.8 & 7.6 & 18 \\
\hline 20060126.3 & 3.368 & 2.581 & 11.5 & 83.5 & 6.9 & 18 \\
\hline 20060126.2 & 3.368 & 2.579 & 11.5 & 83.5 & 6.9 & 18 \\
\hline \multicolumn{7}{|c|}{ (196) Philomela } \\
\hline 20031116.3 & 3.182 & 2.279 & 8.6 & 82.1 & 0.2 & 3 \\
\hline 20031122.3 & 3.183 & 2.244 & 6.6 & 81.1 & 0.3 & 3 \\
\hline 20031123.4 & 3.183 & 2.238 & 6.2 & 80.9 & 0.4 & 3 \\
\hline 20050301.9 & 3.152 & 2.182 & 4.5 & 151.5 & 10.4 & 19 \\
\hline 20050302.8 & 3.152 & 2.184 & 4.7 & 151.3 & 10.4 & 19 \\
\hline 20050304.9 & 3.151 & 2.189 & 5.3 & 150.9 & 10.4 & 19 \\
\hline 20050306.9 & 3.151 & 2.195 & 5.9 & 150.5 & 10.4 & 19 \\
\hline 20060505.0 & 3.061 & 2.068 & 4.1 & 236.2 & 3.7 & 20 \\
\hline 20060507.0 & 3.060 & 2.062 & 3.4 & 235.8 & 3.7 & 20 \\
\hline 20060505.1 & 3.061 & 2.068 & 4.0 & 236.1 & 3.7 & 20 \\
\hline 20060519.0 & 3.059 & 2.050 & 1.8 & 233.4 & 3.3 & 20 \\
\hline 20060519.0 & 3.059 & 2.050 & 1.8 & 233.4 & 3.3 & 20 \\
\hline 20060512.0 & 3.060 & 2.052 & 1.7 & 234.8 & 3.5 & 21 \\
\hline
\end{tabular}


J. Ďurech et al.: Physical models of ten asteroids, Online Material p 4

Table 2. continued.

\begin{tabular}{|c|c|c|c|c|c|c|}
\hline Date & $\begin{array}{c}r \\
{[\mathrm{AU}]}\end{array}$ & $\begin{array}{c}\Delta \\
{[\mathrm{AU}]}\end{array}$ & $\begin{array}{c}\alpha \\
{[\mathrm{deg}]}\end{array}$ & $\begin{array}{c}\lambda \\
{[\mathrm{deg}]}\end{array}$ & $\begin{array}{c}\beta \\
{[\mathrm{deg}]}\end{array}$ & $\begin{array}{l}\text { Obs. } \\
\text { code }\end{array}$ \\
\hline \multicolumn{7}{|c|}{ (218) Bianca } \\
\hline 19960119.1 & 2.676 & 1.776 & 10.4 & 144.5 & -13.7 & 9 \\
\hline 19960318.9 & 2.605 & 1.818 & 16.2 & 132.4 & -8.5 & 8 \\
\hline 19970514.0 & 2.396 & 1.632 & 19.2 & 280.7 & 22.7 & 2 \\
\hline 19970517.0 & 2.398 & 1.609 & 18.5 & 280.6 & 23.0 & 2 \\
\hline 19970519.0 & 2.399 & 1.595 & 18.1 & 280.5 & 23.2 & 2 \\
\hline 19970606.0 & 2.411 & 1.498 & 13.4 & 278.4 & 24.8 & 2 \\
\hline 19970607.0 & 2.412 & 1.494 & 13.1 & 278.2 & 24.8 & 2 \\
\hline 19970607.9 & 2.412 & 1.491 & 12.9 & 278.0 & 24.9 & 2 \\
\hline 20000322.8 & 2.756 & 2.467 & 21.1 & 98.6 & -13.3 & 2 \\
\hline 20000323.8 & 2.755 & 2.479 & 21.1 & 98.8 & -13.2 & 2 \\
\hline 20010302.1 & 2.384 & 1.779 & 22.0 & 225.2 & 11.1 & 2 \\
\hline 20010306.1 & 2.382 & 1.734 & 21.3 & 225.6 & 11.7 & 2 \\
\hline 20010307.1 & 2.381 & 1.723 & 21.1 & 225.6 & 11.9 & 2 \\
\hline 20010401.1 & 2.369 & 1.494 & 14.7 & 225.3 & 16.0 & 2 \\
\hline 20010403.0 & 2.368 & 1.481 & 14.1 & 225.1 & 16.4 & 2 \\
\hline 20031213.9 & 2.949 & 2.026 & 8.0 & 70.5 & -22.3 & 5 \\
\hline 20031215.9 & 2.949 & 2.031 & 8.4 & 70.1 & -22.3 & 5 \\
\hline 20031216.9 & 2.948 & 2.034 & 8.5 & 69.8 & -22.3 & 5 \\
\hline 20031217.8 & 2.948 & 2.037 & 8.7 & 69.6 & -22.2 & 5 \\
\hline 20031220.8 & 2.946 & 2.048 & 9.4 & 68.9 & -22.1 & 5 \\
\hline 20040110.8 & 2.935 & 2.183 & 14.4 & 65.6 & -20.7 & 5 \\
\hline 20040114.8 & 2.932 & 2.219 & 15.3 & 65.2 & -20.3 & 5 \\
\hline 20040117.8 & 2.931 & 2.248 & 15.9 & 65.1 & -20.0 & 5 \\
\hline 20050117.1 & 2.546 & 1.925 & 19.8 & 178.4 & -4.4 & 2 \\
\hline 20050202.0 & 2.527 & 1.736 & 16.1 & 178.5 & -3.4 & 2 \\
\hline 20050207.1 & 2.521 & 1.684 & 14.5 & 178.1 & -3.0 & 2 \\
\hline 20050209.1 & 2.519 & 1.665 & 13.8 & 177.9 & -2.8 & 2 \\
\hline 20050311.6 & 2.485 & 1.491 & 0.4 & 172.0 & 0.3 & 16 \\
\hline 20050312.6 & 2.484 & 1.490 & 0.2 & 171.8 & 0.4 & 16 \\
\hline 20050313.6 & 2.483 & 1.489 & 0.6 & 171.5 & 0.5 & 22 \\
\hline 20050313.6 & 2.482 & 1.489 & 0.7 & 171.5 & 0.5 & 16 \\
\hline 20050326.5 & 2.469 & 1.499 & 7.0 & 168.4 & 2.0 & 16 \\
\hline 20050331.5 & 2.464 & 1.515 & 9.4 & 167.3 & 2.5 & 16 \\
\hline 20050403.4 & 2.461 & 1.527 & 10.7 & 166.8 & 2.8 & 22 \\
\hline 20050416.9 & 2.447 & 1.609 & 16.0 & 164.9 & 4.1 & 23 \\
\hline 20050418.9 & 2.445 & 1.624 & 16.7 & 164.7 & 4.2 & 23 \\
\hline 20050419.9 & 2.444 & 1.632 & 17.0 & 164.6 & 4.3 & 23 \\
\hline 20050406.9 & 2.457 & 1.545 & 12.1 & 166.1 & 3.2 & 23 \\
\hline 20050410.9 & 2.453 & 1.568 & 13.8 & 165.5 & 3.5 & 23 \\
\hline 20050208.4 & 2.520 & 1.671 & 14.1 & 178.0 & -2.9 & 4 \\
\hline 20050211.4 & 2.516 & 1.643 & 13.0 & 177.6 & -2.6 & 4 \\
\hline 20050212.2 & 2.515 & 1.636 & 12.7 & 177.5 & -2.5 & 4 \\
\hline 20050330.1 & 2.465 & 1.510 & 8.7 & 167.6 & 2.4 & 4 \\
\hline 20050405.1 & 2.459 & 1.535 & 11.4 & 166.5 & 3.0 & 4 \\
\hline 20050406.1 & 2.458 & 1.540 & 11.8 & 166.3 & 3.1 & 4 \\
\hline \multicolumn{7}{|c|}{ (306) Unitas } \\
\hline 20030309.2 & 2.700 & 1.849 & 13.2 & 129.8 & 0.2 & 24 \\
\hline 20030310.2 & 2.699 & 1.857 & 13.5 & 129.6 & 0.2 & 24 \\
\hline 20030401.1 & 2.689 & 2.077 & 19.3 & 128.4 & 0.9 & 24 \\
\hline 20030410.2 & 2.684 & 2.184 & 20.7 & 128.8 & 1.2 & 24 \\
\hline \multicolumn{7}{|c|}{ (423) Diotima } \\
\hline 19980924.3 & 3.114 & 2.365 & 14.1 & 49.6 & -8.4 & 28 \\
\hline 19980925.3 & 3.115 & 2.354 & 13.9 & 49.5 & -8.4 & 28 \\
\hline 19980926.3 & 3.115 & 2.344 & 13.6 & 49.5 & -8.4 & 28 \\
\hline 19980928.3 & 3.116 & 2.326 & 13.1 & 49.3 & -8.4 & 28 \\
\hline 19980929.2 & 3.116 & 2.317 & 12.9 & 49.2 & -8.4 & 28 \\
\hline 19981016.2 & 3.122 & 2.194 & 7.9 & 47.0 & -8.1 & 29 \\
\hline 19981028.2 & 3.126 & 2.150 & 4.0 & 44.7 & -7.8 & 29 \\
\hline 19981028.2 & 3.126 & 2.150 & 4.0 & 44.7 & -7.8 & 28 \\
\hline 19981030.1 & 3.127 & 2.146 & 3.5 & 44.3 & -7.7 & 29 \\
\hline 19981030.2 & 3.127 & 2.146 & 3.5 & 44.3 & -7.7 & 28 \\
\hline 19981110.2 & 3.131 & 2.149 & 2.9 & 42.0 & -7.2 & 28 \\
\hline 19981112.2 & 3.131 & 2.153 & 3.4 & 41.6 & -7.1 & 28 \\
\hline 20031016.1 & 3.078 & 2.111 & 5.5 & 11.6 & -13.5 & 13 \\
\hline 20031017.0 & 3.078 & 2.113 & 5.7 & 11.5 & -13.4 & 13 \\
\hline
\end{tabular}


Table 2. continued.

\begin{tabular}{|c|c|c|c|c|c|c|}
\hline Date & $\begin{array}{c}r \\
{[\mathrm{AU}]}\end{array}$ & $\begin{array}{c}\Delta \\
{[\mathrm{AU}]}\end{array}$ & $\begin{array}{c}\alpha \\
{[\mathrm{deg}]}\end{array}$ & $\begin{array}{c}\lambda \\
{[\mathrm{deg}]}\end{array}$ & $\begin{array}{c}\beta \\
{[\mathrm{deg}]}\end{array}$ & $\begin{array}{l}\text { Obs. } \\
\text { code }\end{array}$ \\
\hline 20041207.1 & 3.189 & 2.237 & 5.4 & 92.4 & 4.9 & 4 \\
\hline 20041207.3 & 3.189 & 2.236 & 5.3 & 92.3 & 4.9 & 4 \\
\hline 20041213.2 & 3.190 & 2.218 & 3.4 & 91.1 & 5.2 & 4 \\
\hline 20041213.4 & 3.190 & 2.218 & 3.3 & 91.1 & 5.2 & 4 \\
\hline 20050112.8 & 3.191 & 2.291 & 8.4 & 85.0 & 6.4 & 14 \\
\hline 20050113.0 & 3.191 & 2.291 & 8.5 & 85.0 & 6.4 & 14 \\
\hline 20050131.8 & 3.191 & 2.461 & 13.6 & 82.9 & 6.7 & 2 \\
\hline 20050221.9 & 3.191 & 2.720 & 17.0 & 82.9 & 6.7 & 2 \\
\hline 20050320.8 & 3.190 & 3.099 & 18.1 & 86.2 & 6.7 & 2 \\
\hline 20060117.1 & 3.124 & 2.477 & 15.3 & 172.4 & 14.2 & 2 \\
\hline 20060228.1 & 3.109 & 2.152 & 5.7 & 167.2 & 16.3 & 2 \\
\hline 20060309.1 & 3.106 & 2.141 & 5.2 & 165.3 & 16.3 & 2 \\
\hline 20060313.0 & 3.105 & 2.144 & 5.7 & 164.5 & 16.3 & 2 \\
\hline \multicolumn{7}{|c|}{ (776) Berbericia } \\
\hline 20031124.3 & 2.673 & 1.863 & 14.5 & 104.0 & 4.4 & 3 \\
\hline 20031126.2 & 2.676 & 1.848 & 13.9 & 103.8 & 4.7 & 3 \\
\hline 20040210.8 & 2.796 & 2.078 & 16.1 & 90.2 & 11.2 & 5 \\
\hline 20040212.8 & 2.799 & 2.102 & 16.6 & 90.2 & 11.2 & 5 \\
\hline 20040213.8 & 2.801 & 2.115 & 16.8 & 90.2 & 11.2 & 5 \\
\hline 20040215.9 & 2.804 & 2.141 & 17.2 & 90.2 & 11.3 & 5 \\
\hline 20040219.9 & 2.811 & 2.194 & 17.9 & 90.2 & 11.3 & 5 \\
\hline 20040223.9 & 2.817 & 2.249 & 18.5 & 90.4 & 11.4 & 5 \\
\hline 20040228.9 & 2.826 & 2.319 & 19.1 & 90.7 & 11.4 & 5 \\
\hline 20050215.1 & 3.309 & 2.529 & 12.0 & 184.5 & 24.1 & 21 \\
\hline 20050219.1 & 3.313 & 2.500 & 11.2 & 184.0 & 24.4 & 21 \\
\hline 20050312.0 & 3.330 & 2.412 & 7.7 & 180.0 & 25.3 & 25 \\
\hline 20050313.0 & 3.331 & 2.411 & 7.6 & 179.8 & 25.4 & 25 \\
\hline 20050509.9 & 3.370 & 2.759 & 15.2 & 171.0 & 21.6 & 2 \\
\hline 20060614.2 & 3.276 & 2.335 & 8.0 & 237.3 & 6.8 & 11 \\
\hline 20060615.2 & 3.275 & 2.340 & 8.3 & 237.1 & 6.7 & 11 \\
\hline 20060616.2 & 3.274 & 2.345 & 8.6 & 237.0 & 6.6 & 11 \\
\hline 20060618.2 & 3.272 & 2.357 & 9.1 & 236.6 & 6.4 & 26 \\
\hline 20060619.2 & 3.271 & 2.363 & 9.4 & 236.5 & 6.4 & 11 \\
\hline 20060620.2 & 3.270 & 2.369 & 9.7 & 236.3 & 6.3 & 11 \\
\hline \multicolumn{7}{|c|}{ (944) Hidalgo } \\
\hline 20041014.3 & 2.184 & 1.240 & 11.2 & 40.6 & 16.3 & 24 \\
\hline 20041017.3 & 2.171 & 1.221 & 10.6 & 39.8 & 17.8 & 24 \\
\hline 20041018.3 & 2.166 & 1.215 & 10.4 & 39.5 & 18.4 & 24 \\
\hline 20041021.4 & 2.153 & 1.200 & 10.2 & 38.7 & 20.0 & 24 \\
\hline
\end{tabular}

Table 3. The table lists the ecliptic coordinates of the asteroid's spin axis direction $\left(\lambda_{\mathrm{p}}, \beta_{\mathrm{p}}\right)$, its sidereal rotation period $P$, the span of observations in years, the number of oppositions $N_{\text {opp }}$, the number of lightcurves $N_{\mathrm{lc}}$, and the rms residual of the fit.

\begin{tabular}{lrrccccc}
\hline \hline Asteroid & $\begin{array}{c}\lambda_{\mathrm{p}} \\
{[\mathrm{deg}]}\end{array}$ & $\begin{array}{c}\beta_{\mathrm{p}} \\
{[\mathrm{deg}]}\end{array}$ & $\begin{array}{c}P \\
{[\mathrm{hr}]}\end{array}$ & Years of obs. & $N_{\text {opp }}$ & $N_{\text {lc }}$ & $\begin{array}{c}\text { rms } \\
{[\mathrm{mag}]}\end{array}$ \\
\hline (110) Lydia & 331 & -61 & 10.92580 & $1958-2003$ & 4 & 26 & 0.011 \\
& 149 & -55 & & & & & \\
(125) Liberatrix & 280 & +74 & 3.968199 & $1981-2005$ & 7 & 34 & 0.024 \\
& 95 & +68 & & & & & \\
(130) Elektra & 65 & -88 & 5.224664 & $1980-2003$ & 11 & 54 & 0.013 \\
(165) Loreley & 346 & +29 & 7.22667 & $1981-2006$ & 6 & 29 & 0.015 \\
(196) Philomela & 276 & -49 & 8.332827 & $1964-2006$ & 8 & 27 & 0.013 \\
& 111 & -41 & & & & & \\
(218) Bianca & 305 & +17 & 6.33717 & $1979-2005$ & 10 & 50 & 0.015 \\
& 121 & -10 & & & & & \\
(306) Unitas & 79 & -35 & 8.73875 & $1979-2003$ & 5 & 15 & 0.015 \\
& 254 & -18 & & & & & \\
(423) Diotima & 351 & +4 & 4.775377 & $1981-2006$ & 10 & 48 & 0.017 \\
(776) Berbericia & 347 & +12 & 7.66701 & $1977-2006$ & 8 & 36 & 0.012 \\
(944) Hidalgo & 281 & +5 & 10.058634 & $1976-2004$ & 4 & 14 & 0.013 \\
\hline
\end{tabular}

